Dom. Cien., ISSN: 2477-8818

Vol. 5, núm.1., ene, 2019, pp. 459-487

\title{
El cambio climático y su incidencia en enfermedades tropicales
}

\section{Climate change and its incidence in tropical diseases}

\section{Mudança climática e sua incidência em doenças tropicais}

\author{
Nakin A. Véliz-Mero ${ }^{\text {I }}$ \\ nakin.veliz@uleam.edu.ec \\ Nidia E. Macías-Cedeño II \\ necmacias@utm.edu.ec \\ José M. Piguave-Reyes III \\ jose.manuel.piguave@hotmail.com
}

\author{
Yessenia L. Moreira-Andrade IV \\ ylmoreira@utm.edu.ec \\ Fabrizzio R. Araujo-Reyna ${ }^{\mathrm{V}}$ \\ fabrizzio.araujo@gmail.com \\ Mariela P. Sabando-Saltos VI \\ mpsabando@utm.edu.ec
}

Recibido: 27 de noviembre de 2018 * Corregido: 18 de diciembre de $2018 *$ Aceptado: 20 de diciembre de 2018

I. Doctor en Medicina y Cirugía; Magister en Gerencia de Salud para el Desarrollo Local; Magister en Gestión y Desarrollo Social; Universidad Laica Eloy Alfaro de Manabí, Manta, Ecuador.

II. Licenciada en Enfermería; Especialista en Gerencia en Salud; Universidad Técnica de Manabí, Jipijapa, Ecuador.

III. Licenciado en Laboratorio Clínico; Magister en Epidemiología; Centro Especializado en Diagnóstico y Tratamiento Muñoz, Quito, Ecuador.

IV. Magister en Gerencia de Salud; Hospital Napoleón Dávila Córdova; Universidad Técnica de Manabí, Jipijapa, Ecuador.

v. Magister en Epidemiologia; Diplomado en APS; Médico General; Epidemiólogo Hospital de Infectología Guayaquil, Guayaquil, Ecuador.

VI. Licenciada en Enfermería; Magíster en Gerencia en Salud; Universidad Técnica de Manabí, Jipijapa, Ecuador. 


\section{Resumen}

El desarrollo de las sociedades a ayudado a generar nuevas tecnologías en busca de minimizar las necesidades de la humanidad. Estos cambios han generado efectos colaterales produciendo daños al ecosistema y por ende disminución de la calidad de vida de las sociedades. Unos de los principales problemas a los cuales se ha visto afectado es la salud y sobre todo en esta parte del continente debido a los factores socioculturales y económicos que no han permitido un avance en esta materia. El objetivo general de esta investigación es analizar el efecto del cambio climático y su incidencia en enfermedades tropicales. La metodología utilizada se basó en una investigación de campo con carácter descriptivo, explicativo y exploratorio. Los resultados se desglosaron en base a el cambio climático y la salud, los tipos de enfermedades que provienen del cambio climático y como mitigar las enfermedades tropicales producto del cambio climático. Como conclusión se determinó que se debe producir un quiebre en los paradigmas de las sociedades y poder afianzar una nueva cultura ecológica que permita solventar el cambio climático, pero también contrarrestar las enfermedades que son producto de este terrible problema global.

Palabras claves: Sociedad; Cambio Climático; Enfermedades Tropicales; Cultura Ecológica. 


\begin{abstract}
The development of societies has helped to generate new technologies in order to minimize the needs of humanity. These changes have generated collateral effects producing damage to the ecosystem and therefore a reduction in the quality of life of societies. One of the main problems to which has been affected is health and especially in this part of the continent due to socio-cultural and economic factors that have not allowed an advance in this area. The general objective of this research is to analyze the effect of climate change and its incidence on tropical diseases. The methodology used was based on a field investigation with descriptive, explanatory and exploratory character. The results were disaggregated on the basis of climate change and health, the types of diseases that come from climate change and how to mitigate tropical diseases caused by climate change. As a conclusion, it was determined that a break in the paradigms of societies should be produced and a new ecological culture could be established to solve climate change, but also to counteract the diseases that are the product of this terrible global problem.
\end{abstract}

Key words: Society; Climate Change; Tropical Diseases; Ecological Culture. 


\section{Introducción.}

El desarrollo de las capacidades del ser humano va de la mano con la necesidad de poder satisfacer las necesidades básicas de la sociedad. El proceso evolutivo va enmarcado hacia esa dirección. El crecimiento poblacional ha hecho de que la sociedad busque miles de formas de poder mantener a esta gran población. Con el desarrollo de la revolución industrial empezó a satisfacer esas necesidades a corto tiempo ya que los procesos y sistemas ayudaban a generar tal efecto. Pero al cabo de unos cuantos y largos años, se empezó a evidenciar que el avance tecnológico, en busca del bienestar global del hombre, genero un efecto colateral dentro del ambiente. Es aquí donde entra en juego el cambio climático.

El mundo empezó a tener la necesidad de poder atacar este problema que ha afectado la vida de las sociedades ocasionando problemas de subsistencia. El cambio climático se ha convertido en uno de los grandes temas de la política pública mundial y nacional durante los últimos quince años (Barton, 2009).

El cambio climático debido a actividades humanas compromete ahora la sostenibilidad del desarrollo humano en el planeta porque amenaza los sistemas de apoyo ecológico de los que dependen la vida, la salud y el bienestar de la humanidad, cuya continua mejora debe ser la meta primordial del proceso de desarrollo propiamente dicho. (Martens, 1998, pág. 100)

Por supuesto está en juego la sostenibilidad del bienestar de la sociedad a consecuencia del desarrollo de nuevas tecnologías. Lo que resulta más difícil de probar es que la revolución tecnológica que provoca una parte de la humanidad y ala que asiste expectante el resto de la misma sea la causante del deterioro del clima (Sanz Donaire, 1999).

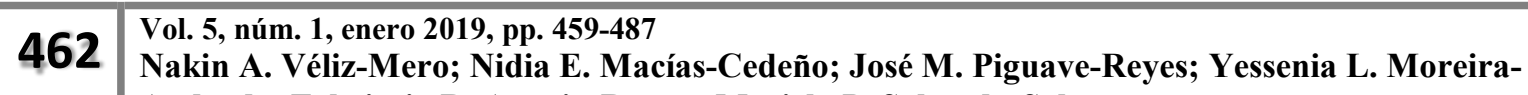
Andrade; Fabrizzio R. Araujo-Reyna; Mariela P. Sabando-Saltos
} 
Ahora poder determinar el deterioro del clima es importante para poder contrarrestar este fenómeno por lo cual es necesario poder medir los gases invernaderos y su influencia en el cambio climático. En las proyecciones de cambio climático del Panel Intergubernamental sobre Cambio Climático (IPCC), se suele tener en cuenta únicamente la influencia ejercida sobre el clima por los aumentos antropógenos de los gases de efecto invernadero y por otros factores relacionados con los seres humanos (Cueva, 2014). En la Tabla 1 se puede detallar los distintos gases de efecto invernadero.

Tabla 1. Tipos de gases de efecto invernadero

\begin{tabular}{cc}
\hline Descripción del Gas & Formula molecular \\
\hline Dióxido de Carbono & $\mathrm{CO}_{2}$ \\
\hline Metano & $\mathrm{CH}_{4}$ \\
\hline Óxido nitroso & $\mathrm{N}_{2} \mathrm{O}$ \\
\hline Hidrofluorocarbonos & $\mathrm{HFC}$ \\
\hline Perfluorocarbonos & $\mathrm{PFC}$ \\
\hline Hexafluoruro de azufre & $\mathrm{SF}_{6}$ \\
\hline
\end{tabular}

Fuente: (Cueva, 2014)

Estos gases invernadero producen alteraciones en el clima que perjudican la vida de la humanidad lo que conlleva a interpretar el significado de cambio climático el cual sufre confusión con variabilidad climática (VC), el efecto invernadero o calentamiento global. La VC, se refiere a variaciones en las condiciones climáticas medias, y otras estadísticas del clima (como las desviaciones típicas y los fenómenos extremos, entre otras), en todas las escalas temporales y

\footnotetext{
463 Vol. 5, núm. 1, enero 2019, pp. 459-487 
espaciales que se extienden más allá de la escala de un fenómeno meteorológico en particular (QUINTERO, CARVAJAL, \& ALDUNCE, 2012).

El primer concepto clave es el de sistema climático. Décadas atrás cuando se hablaba del clima, sólo se pensaba en la atmósfera, siendo, sucintamente, su estado estructural, estable en una escala de tiempo algo dilatada (multidecadal, secular, milenaria y aún más), a diferencia del tiempo meteorológico, que es su comportamiento coyuntural, efímero, cambiante, en escalas de tiempo cortas (diaria, por ejemplo). La atmósfera era, pues, el objeto casi exclusivo del análisis del tiempo y del clima. Sin embargo, desde hace algunas décadas ha surgido como nuclear en el estudio del clima presente y futuro el concepto de sistema climático. (Martín Vide, 2009, pág. 52)

Las observaciones empíricas sugieren que el calentamiento del sistema climático es una realidad y se caracteriza por:

$\checkmark$ Incremento de la temperatura global: Según la NASA; la temperatura promedio global superficie-océano aumentó en promedio $0,01^{\circ} \mathrm{C}$ al año en el período 1900-2007. Así, al 2007 la temperatura global promedio habría aumentado en $0.66^{\circ} \mathrm{C}$ respecto al año 1900 .

$\checkmark$ Aumento del nivel de los océanos mundiales: 2 milímetros promedio al año en el período 1961-2003.

Deshielo generalizado de nevados, glaciares y mantos polares: Las mediciones satelitales revelan que los glaciares de Groenlandia y la Antártida están perdiéndose a un ritmo de 125 mil millones de toneladas al año.

\footnotetext{
464 Vol. 5, núm. 1, enero 2019, pp. 459-487 Nakin A. Véliz-Mero; Nidia E. Macías-Cedeño; José M. Piguave-Reyes; Yessenia L. MoreiraAndrade; Fabrizzio R. Araujo-Reyna; Mariela P. Sabando-Saltos
} 
$\checkmark$ Mayor variabilidad de las precipitaciones: El calentamiento global produce una mayor evaporación de la superficie del océano, intensificando el ciclo hidrológico y aumentando las precipitaciones de manera variable. Así, se prevé que aumentarán en latitudes altas y disminuirán en las bajas. (Vargas, 2009, pág. 13)

De aquí entonces es necesario poder definir el cambio climático con el fin de poder aplicar el método, técnicas e instrumentos que permitan disminuir este efecto en la calidad de vida de las sociedades.

El cambio climático ha de entenderse una variación con una significación estadística clara en períodos de tiempo algo dilatados, decadales o superiores, en los parámetros de tendencia central y/o de dispersión de al menos una variable climática (temperatura, precipitación, etc.) en el conjunto del planeta o claramente visible en ámbitos continentales o superiores. (Martín Vide, 2009, pág. 55)

Esto sugiere un cambio en la perspectiva de la humanidad en la cual debe ir en busca de poder hacer cambios en el estilo de vida, con el cual tendrá efecto en el proceso productivo y por ende cambios políticos dentro de las comunidades. El entendimiento de las consecuencias del cambio climático se está traduciendo en el planteo, tanto a nivel académico, político como empresario, del crecimiento verde, que no sólo contempla medidas de mitigación y de adaptación, sino que constituye una alternativa al presente patrón de crecimiento económico (Bianchi, 2016).

Este cambio de conducta dentro de las poblaciones ha permitido extenderse en cada una de las organizaciones que conforman las sociedades lo cual conlleva a que las organizaciones empresariales lleven la batuta de este cambio. Han implementado una serie de políticas para mitigar 
este flagelo al clima. Se han creado normas o estatutos que permiten determinar los pasos o pautas para la elaboración de productos sin la generación de la contaminación ambiental. Esto se puede observar en la Figura 1 donde se detalla el esquema general de innovaciones ambientales en una empresa.

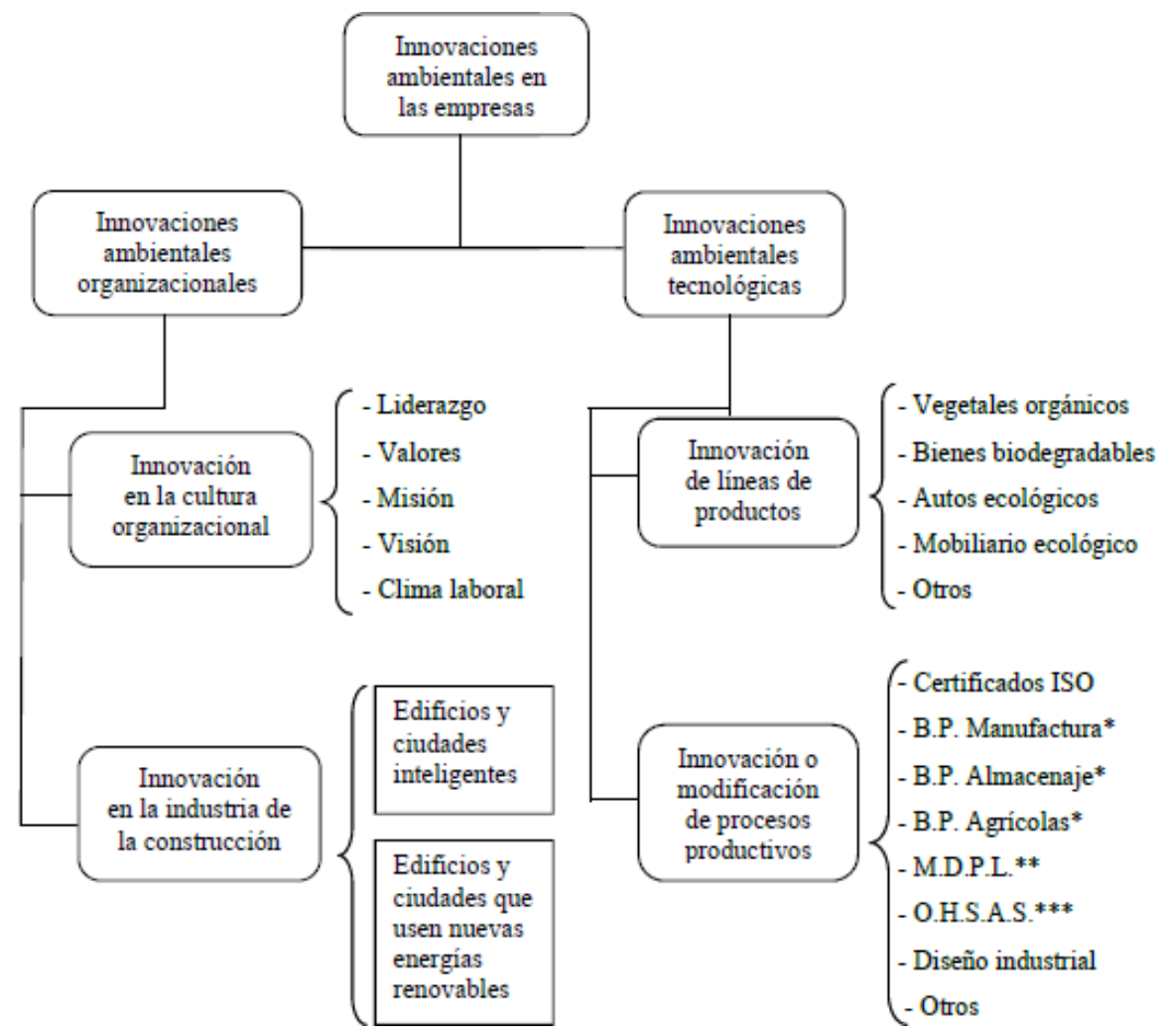

Figura 1. Esquema general de innovaciones ambientales en una empresa

Fuente: (Cueva, 2014)

En el Ecuador se ha implementado varios procesos legislativos ambientales que promulgan leyes en materia ambiental en búsqueda de relacionar el cambio climático. Además, ha establecido instrumentos de gestión y manejo sostenible de los ecosistemas, mediante cambios administrativos y 
aplicación de la legislación en actividades gubernamentales, a fin de generar la estabilidad que requieren las políticas ambientales de Estado (Sánchez L. W., 2015).

Estos planes estratégicos del gobierno del Ecuador deben evaluar las situaciones geográficas de la nación con las actividades que combatan el cambio climático. Un ejemplo es en el páramo ya que se podrían haber comparado la opción de la forestación, con la opción de introducir sistemas agroforestales en los cultivos campesinos, y también se podría haber comparado con una opción de regeneración natural del bosque andino (Vidal, 1999).

Indudablemente el cambio climático ha jugado un papel importante en el cambio de vida de las sociedades, impulsadas por el deterioro de su calidad de vida por el constante auge de la tecnología. Es necesario poder determinar los factores o causas que impulsan este crecimiento del cambio climático.

Los factores que afectan los cambios de temperatura media de la tierra y el cambio climático son los cambios en el desnivel del mar, los efectos de las nubes, la emisión de aerosoles a la atmósfera, aumento en las emisiones de dióxido de carbono, gas metano, hidratos de metano. Además, los cambios de reflexión terrestres y los cambios en el campo magnético exterior. Existen otros factores son la contaminación del aire, los cambios en el hielo polar, el contenido en vapor de agua y la cantidad de cobertura de nubes y la cantidad de energía solar que alcanza la Tierra. (Díaz Cordero, 2012, pág. 231)

Como se determinó que uno de los principales causantes de este cambio climático son los gases de efecto invernadero (GEI) entonces es necesario poder determinar cuál es la fuente de dichos gases. Las fuentes son la actividad industrial, el sector energía, y, en menor medida, la

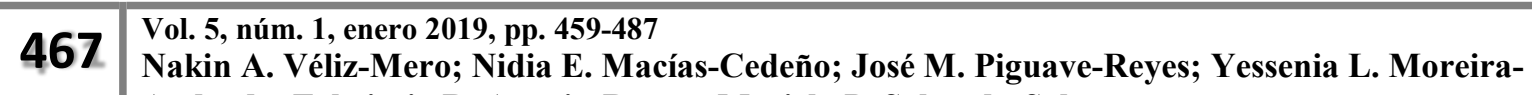
Andrade; Fabrizzio R. Araujo-Reyna; Mariela P. Sabando-Saltos
} 
actividad agropecuaria. Sin embargo, la quema de biomasa en la agricultura y en los bosques también disminuye la captura de carbono a través de la fotosíntesis (Vargas, 2009). Esto se puede observar en la Figura 2.

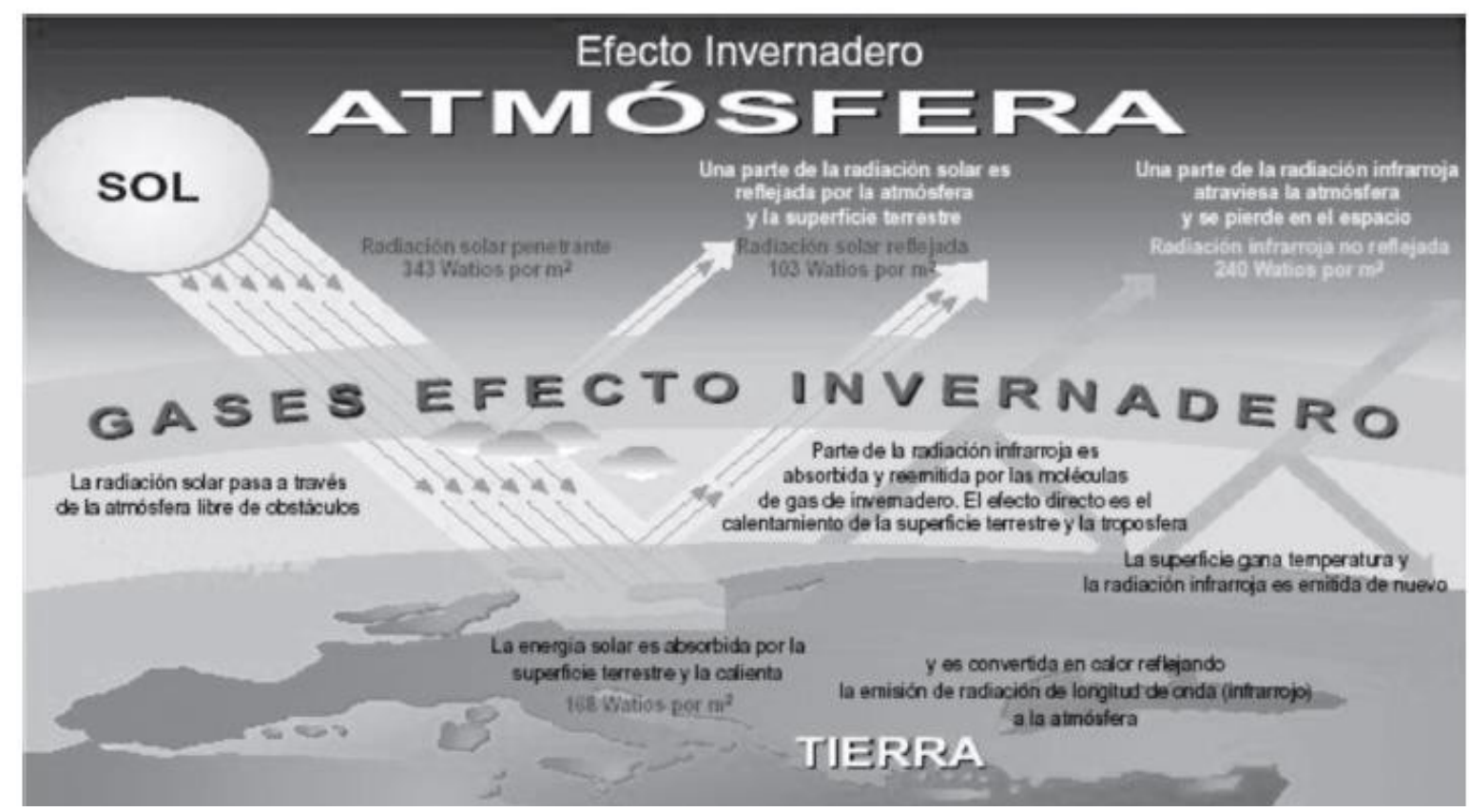

Figura 2. Efecto Invernadero

Fuente: (Berberian \& Rosanova, 2012)

Estos gases ocasionan que tanto la superficie de la Tierra como la parte inferior de la atmósfera se calienten y, sin éstos, "la temperatura media de la Tierra debería ser $20^{\circ} \mathrm{C}$ bajo cero [...] La tierra debería ser un lugar hostil para casi todas las formas de vida”. (Butze Aguilar, 2004, pág. 67)

Esto requiere la comprensión del cambio climático para poder combatir los problemas que ocasiona al ecosistema y al brusco cambio del estilo de vida de la humanidad. La vulnerabilidad al cambio climático no permite esta simplificación, ya que existe una discrepancia entre la escala 
física, en la que se manifiesta la amenaza (que es global), y la escala en la que se analiza la exposición (regional o local) (Lampis, 2013).

La existencia de problemas ambientales (no problemática, ni crisis), cuyo origen se puede atribuir a la acción humana, nadie la puede negar. Pero son problemas de ajuste - daños colaterales- y, en todo caso, la historia demuestra que son resolubles gracias al ingenio humano y a su plasmación en alternativas sociales y tecnológicas que mejoran continuamente los procesos de obtención, transformación y distribución de los recursos naturales. (Meira Cartea, 2013, pág. 31)

Uno de estos problemas colaterales producto del cambio climático son los desplazados ambientales. Denominar a las personas que se desplazan por causas medioambientales no solo favorece la confusión, sino que limita el reconocimiento del fenómeno como un tipo de migración forzada, el cual requiere de una protección especial por parte de los Estados y de la comunidad internacional (Egea Jiménez, 2011).

Toda persona que se ha visto forzada a abandonar su hábitat tradicional, temporal o permanentemente, debido a una disrupción medioambiental marcada (natural o desencadenada por el Hombre), que compromete su existencia y/o afecta seriamente su calidad de vida (...) Se entiende en esta definición por "disrupción medioambiental” cualquier cambio físico, químico y/o biológico en el ecosistema (o recurso de base), que lo convierte en inutilizable, temporal o permanentemente, para sustentar la vida humana. (HINNAWI, 1985, pág. 71)

La economía mundial se ha estado afectada por las constantes regulaciones por parte de los estados a las empresas y porque estas últimas deben cambiar su metodología de producción, haciéndose más amigables al medio ambiente. El comercio internacional se ha visto influido por el

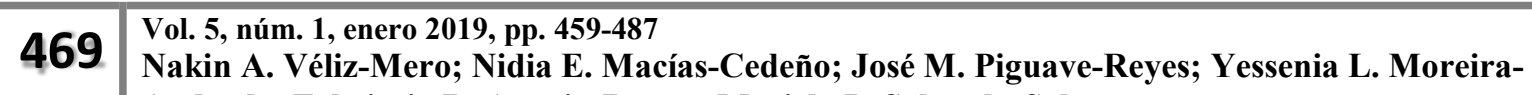
Andrade; Fabrizzio R. Araujo-Reyna; Mariela P. Sabando-Saltos
} 
cambio climático, pero también éste influye en él, desde el abastecimiento de los insumos, la producción, el transporte, la venta, el consumo de los productos y cómo se reciclan los desechos (Cueva, 2014).

También la agricultura ha tenido cambios producto a que es uno de los principales causantes de los gases del efecto invernadero. Esta relación con la sociedad ha impulsado un sin fin de normas y leyes que permita apaciguar la emisión de estos gases a la atmosfera. Está claro que el sector debe contribuir tanto al esfuerzo para controlar las emisiones como prepararse para la adaptación para enfrentarse a los riesgos y vulnerabilidades del cambio climático (Iglesias, 2009).

Poder mitigar este problema de cultivos que utilizan fertilizantes altamente contaminantes permite buscar soluciones en todos los ámbitos de la sociedad. Se ha planteado soluciones tradicionales infundidas por nuestros primeros habitantes, los indígenas.

Las poblaciones indígenas prevén y reaccionan a las crisis ambientales en formas basadas en su conocimiento ecológico tradicional. Por ejemplo, una estrategia usada comúnmente para minimizar el riesgo asociado a potenciales crisis ambientales (sequía, inundación) es el uso de la diversidad. Numerosas comunidades indígenas utilizan sistemáticamente una gran diversidad de cultivos, de variedades de cultivos y hasta de ecosistemas con el objetivo no de maximizar los ingresos sino de minimizarlos. Al usar diferentes variedades, en caso de acontecimientos extremos (sequía), al menos se obtendrá el cultivo de las variedades más tolerantes a este acontecimiento. El uso de la diversidad en nuestros sistemas productivos puede ayudarnos a minimizar riesgos ante la creciente variabilidad climática. (Reyes-García, 2007, págs. 115-116)

\footnotetext{
\begin{tabular}{l|l}
470 & Vol. 5, núm. 1, enero 2019, pp. 459-487 \\
Nakin A. Véliz-Mero; Nidia E. Mac
\end{tabular} Nakin A. Véliz-Mero; Nidia E. Macías-Cedeño; José M. Piguave-Reyes; Yessenia L. MoreiraAndrade; Fabrizzio R. Araujo-Reyna; Mariela P. Sabando-Saltos
} 
Otra problemática que se debe estudiar y analizar es la planificación de ciudades y regiones las cuales deben mermar los resultados del cambio climático. Esto debe ser integrado como un aspecto central, no como algo adicional o complementario. Este tipo de planificación ha sido usada en forma constante durante las últimas décadas para otros desafíos de política pública como género, medio ambiente y diversidad cultural (Barton, 2009).

Pero todos estos problemas se hacen mínimos comparados con la incidencia en la salud de la población.

El impacto del cambio climático sobre la salud humana se explica en gran parte por los cambios demográficos. Los peores escenarios climáticos tendrán lugar en las zonas más desfavorecidas y pobladas. Así, el crecimiento de la población interferirá con el aumento de la desertización y la consiguiente falta de alimentos y de agua, la superpoblación de las zonas costeras inundables y la migración masiva hacia las grandes urbes. (Sunyer, 2010, pág. 101)

Los efectos a la salud provocados por el cambio climático precisan de nuevas estrategias para mitigarlos, con enfoque multidisciplinario e intersectorial, donde las acciones de prevención y promoción de salud son imprescindibles en el abordaje de esta temática. (González Sánchez \& Gutiérrez Soto, 2013).

Por lo tanto, esta investigación tiene como objetivo general analizar el efecto del cambio climático y su incidencia en enfermedades tropicales. La metodología utilizada se basó en una investigación decampo especificando los métodos descriptivos, explicativos y exploratorios tomando como base libros, artículos científicos, revistas científicas y documentos web.

\footnotetext{
\begin{tabular}{l|l} 
Vol. 5, núm. 1, enero 2019, pp. 459-487 \\
Nakin A. Véliz-Mero; Nidia E. Macias
\end{tabular} acías-Cedeño; José M. Piguave-Reyes; Yessenia L. MoreiraAndrade; Fabrizzio R. Araujo-Reyna; Mariela P. Sabando-Saltos
} 


\section{Método.}

El desarrollo de esta investigación se basó fundamentalmente en una investigación de campo donde se explora los métodos descriptivos, explicativos y exploratorios. La metodología se basó en técnicas basadas en revisión de textos, documentos y artículos científicos publicados disponibles en la web. Aunado se siguió un plan desarrollando y analizando el efecto del cambio climático, la relación del cambio climático y la salud y los efectos del cambio climático sobre las enfermedades tropicales.

\section{Resultados.}

\section{El cambio climático y la salud}

La salud de la población como "estado sostenible" exige el continuo apoyo de actividades que permitan tener aire puro, agua limpia, suficientes alimentos, una temperatura tolerable, un clima estable, protección contra la radiación ultravioleta solar y altos grados de diversidad biológica (Martens, 1998).

Las sociedades menos privilegiadas serán las más susceptibles a este cambio climático. Los estados deben aplicar políticas que ayuden a mitigar esta gran problemática. Los pueblos y los ecosistemas deberán adaptarse a los futuros regímenes climáticos. La infraestructura física de los servicios de salud será afectada, en particular, por el aumento del nivel del mar y por la presencia de eventos climáticos extremos (Feo, y otros, 2009).

Esto se puede reflejar en el impacto que tiene en la salud y en la alteración del ecosistema y que son más propensos en países en vías de desarrollo, tal como lo muestra la Figura 3.

\footnotetext{
472 Vol. 5, núm. 1, enero 2019, pp. 459-487
Nakin A. Véliz-Mero; Nidia E. Macías-Cedeño; José M. Piguave-Reyes; Yessenia L. MoreiraAndrade; Fabrizzio R. Araujo-Reyna; Mariela P. Sabando-Saltos
} 


\begin{tabular}{|c|c|}
\hline $\begin{array}{l}\text { Cambio } \\
\text { Climático }\end{array}$ & $\begin{array}{l}\text { Alteración de } \\
\text { Ecosistemas }\end{array}$ \\
\hline $\begin{array}{l}\text { - Aumenta la temperatura } \\
\text { global } \\
\text { - Se derriten los glaciares } \\
\text { - Sube el nivel de mares y } \\
\text { océanos } \\
\text { - Se incrementa la frecuencia } \\
\text { e intensidad de eventos } \\
\text { meteorológicos }\end{array}$ & $\begin{array}{l}\text { - Olas de calor y frío } \\
\text { - Deterioro de la disponibilidad y } \\
\text { calidad del agua } \\
\text { - Desastres naturales: huracanes, } \\
\text { incendios, inundaciones, sequias } \\
\text { - Escasez y contaminación de } \\
\text { alimentos } \\
\text { - Enrarecimiento del aire } \\
\text { - Cambio en el comportamiento de } \\
\text { vectores }\end{array}$ \\
\hline
\end{tabular}

\begin{tabular}{|l|}
\hline \multicolumn{1}{|c|}{$\begin{array}{c}\text { Impacto sobre } \\
\text { la Salud }\end{array}$} \\
\hline - Deshidratación \\
- Afecciones cardiovasculares \\
- Desnutrición y cuadros carenciales \\
- Emergencia y reemergencia de \\
enfermedades infecciosas de \\
transmisión vectorial \\
- Diarreas y enfermedades transmitidas \\
por alimentos y agua \\
- Enfermedades respiratorias: IRA, \\
asma, alergias. \\
- Cuadros dermatológicos \\
- Cáncer y enfermedades \\
degenerativas \\
- Compromiso de la salud mental \\
\end{tabular}

\section{Figura 3. Consecuencias del cambio climático sobre la salud y el medio ambiente}

Fuente: (Feo, y otros, 2009)

$\checkmark$ Aumento de los impactos en la salud de los eventos climáticos extremos (olas de calor y de frío, inundaciones, sequías, fuegos, etc.).

$\checkmark$ Incremento de la frecuencia de las enfermedades respiratorias debido a los cambios en la calidad del aire y la distribución del polen.

$\checkmark$ Aumento de la incidencia de las enfermedades de origen alimentario y transmitido por el agua, particularmente en las áreas donde los sistemas de saneamiento son precarios.

$\checkmark$ Cambio en la distribución de las enfermedades infecciosas o de sus vectores, y contribución al posible establecimiento de especies tropicales o subtropicales.

Desplazamiento de poblaciones debido al deterioro medio ambiental, la erosión costera, las inundaciones y la pérdida de tierras de cultivo. (Blázquez, 2010, pág. 79) 
Poder controlar este efecto colateral requiere de un compromiso de toda la sociedad. Los médicos juegan un papel importante en el cambio que se debe dar a la problemática de salubridad que lleva estos fenómenos ambientales.

Tal como mencionaba la asamblea de la OMS en 2008, el papel de los profesionales de la salud es importante como catalizadores para defender que la salud debe ser uno de los motores para establecer las políticas que lleven a mitigar las emisiones, así como para velar por el establecimiento de sistemas de información apropiados para evaluar los riesgos en la salud y las diferencias en la vulnerabilidad, predecir los efectos y estimar los costes, promoviendo programas específicos para los países en desarrollo a partir de la cooperación global y estimulando la acción de ámbito local. (Sunyer, 2010, pág. 102)

Esto unido a otros mecanismos que ayuden a controlar la proliferación de enfermedades, pero sobre todo a influir una nueva cultura de salud para las sociedades. Una alternativa, que puede ser complementaria a la anterior, consiste en llevar a cabo evaluaciones periódicas del impacto en salud de la contaminación atmosférica y su posible relación con el cambio climático (Ballester, 2005). En la figura 4 se puede observar las medidas para minimizar el impacto del cambio climático sobre la salud. 
- Establecimiento de un sistema de vigilancia de la situación atmosférica (incluyendo meteorología y pólenes y esporas) y su impacto en salud.

- Medidas legislativas que establezcan estándares y objetivos de calidad del aire.

- Disminución de emisiones de gases contaminantes.

- Puesta en marcha de estrategias en sectores clave (transporte, industrial, urbanismo, vivienda)

- Uso eficiente de la energía

- Utilización progresiva de energías renovables

- Puesta en marcha de acciones para evitar incendios forestales

- Acciones de educación para la salud y promoción de hábitos respetuosos con el medio ambiente

- Fomentar la participación ciudadana

Figura 4. Medidas encaminadas a minimizar el impacto del cambio climático sobre la salud

Fuente: (Ballester, 2005)

En la Figura 5 se puede detallar las opciones para mitigar, moderar o adaptarse a ese efecto del cambio climático.

Existen muchos acuerdos o tratados que buscan disminuir este flagelo creciente en la población menos favorecida. Estos tratados pretenden infundir políticas globales en lucha contra los efectos colaterales del cambio climático.

Las Metas de Desarrollo del Milenio constituyen una guía para la visión de la salud en el presente siglo y brindan sostén a muchos conceptos claves, políticas y estrategias recomendados en la Agenda 21. Los principios operativos son compatibles con el desarrollo sostenible centrado en el ser humano y otorgan prioridad a la acción y la asociación intersectorial. Los gobiernos que ejecuten políticas con una fuerte orientación equitativa serán los que mayor probabilidad tendrán

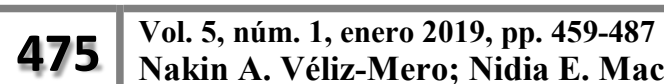




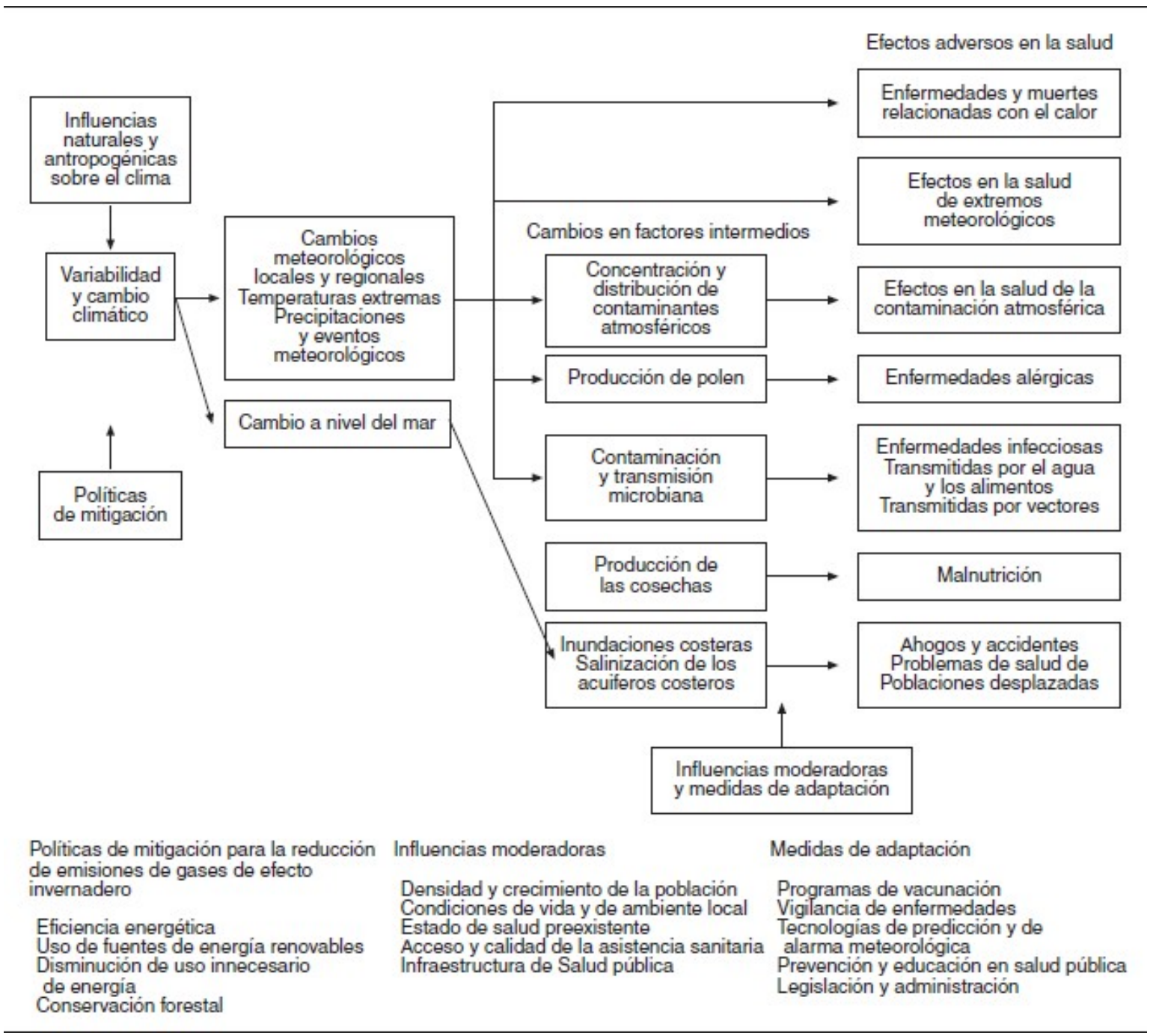

\section{Figura 5. Efectos probables del cambio climático en la salud y posibles respuestas}

Fuente: (Martens, 1998) 
Tipos de enfermedades que provienen del cambio climático

Se reconocen cinco áreas bioclimáticas: glacial, templada, cálida, árida y tropical, agrupándose en las tres últimas la gran mayoría de las enfermedades tropicales. De ahí su nombre. Estas enfermedades son, básicamente, de tres tipos: infecciosas y nutricionales, y muy lejos, genéticas (Pecoul, 2014). Desde el punto de vista geográfico las enfermedades tropicales son las que se manifiestan en esta parte del mundo. Estas enfermedades se estrechan con el tema de la salud.

1) Son características de países en desarrollo como resultado y a la vez causa secundaria del subdesarrollo; 2) cuando se enfocan por género, ponen de relieve la inequidad social que margina, limita y oprime a la mujer, sobre todo a la más pobre, y 3) se proyectan en la organización política y las relaciones de producción como un paño de fondo que determina el subdesarrollo, la enfermedad y la desigualdad. (Pinto Días, 1996, pág. 261)

A pesar de estas políticas globales en la lucha contra las enfermedades que surgen de estos cambios climáticos siguen apareciendo enfermedades que se creían estaban extintas. Se necesita profundizar no sólo en lo relacionado con el paludismo, sino también con otras enfermedades infecciosas y trasmitidas por vectores, como el dengue, la leishmaniasis y la enfermedad de Chagas. (Pabón \& Nicholls, 2005). El impacto climático sobre las enfermedades infecciosas está principalmente relacionado al comportamiento humano, efectos sobre el patógeno y el vector que provocan la enfermedad (Berberian \& Rosanova, 2012).

Estos son solo algunos ejemplos de cómo la interacción entre los pueblos, el intercambio de información, los enfrentamientos bélicos, las nuevas formas de transporte y la construcción de vías de acceso, permiten la difusión de las epidemias, la persistencia y resistencia de ciertos

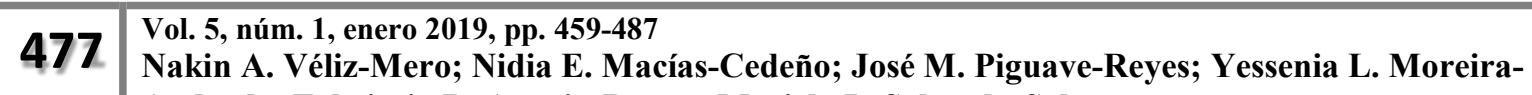
Andrade; Fabrizzio R. Araujo-Reyna; Mariela P. Sabando-Saltos
} 
microorganismos (Valero-Bernal \& Tanne, 2008). También pueden influenciar en la epidemiología de las enfermedades vectoriales: composición atmosférica, urbanización, desarrollo económico y social, comercio internacional, migraciones humanas, desarrollo industrial, uso de la tierra regadíosdesarrollo agrícola (López-Vélez \& Molina Moreno, 2005).

Pero se debe evitar que estos factores aumenten y para ello entran el cambio de cultura de la sociedad y como este parámetro puede influir en la disminución de este impacto en la salud. La progresiva resistencia a insecticidas y medicamentos, deforestación, cambios en políticas públicas sanitarias (reducción de vigilancia, prevención y control vectorial), cambios demográficos (crecimiento poblacional, migraciones, urbanización) y cambios en las condiciones habitacionales (disponibilidad de redes de alcantarillado) (Cerda, Valdivia, Valenzuela, Teresa, \& Venegas, 2008).

Esto invita a que en las políticas públicas la salud sea un elemento central a considerar, por lo que el sector debe tener la fortaleza científica y técnica, así como la capacidad de diálogo y negociación para poder llegar a consensos en donde se vean representados todos los intereses asociados a necesidades bien definidas. Lo anterior se logrará a través de la coordinación interinstitucional e intersectorial. (Moreno, 2010, pág. 169)

Si no se controla estos focos de enfermedades entonces el crecimiento de enfermedades crecerá de manera exponencial. El cambio climático perturba los ecosistemas naturales y favorece las condiciones ideales para la propagación de las infecciones y epidemias, principalmente relacionadas a vectores, agua y alimentos (Berberian \& Rosanova, 2012).

Existe una relación entre los cambios climáticos y las enfermedades de agentes patógenos que han sido reconocidos.

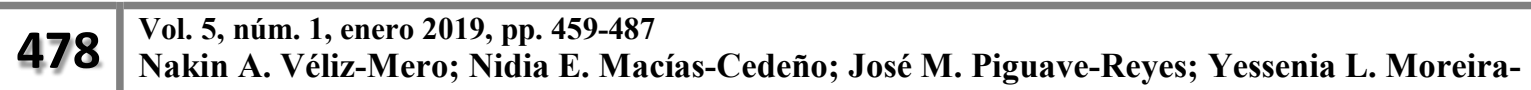
Andrade; Fabrizzio R. Araujo-Reyna; Mariela P. Sabando-Saltos
} 
El efecto de factores climáticos sobre dinámica de población de hospedero parásito es particularmente evidente en las latitudes del norte donde la ocurrencia y la transmisión de parásitos son reguladas por los cambios estacionales, fundamentalmente por los cambios bruscos de la temperatura ambiental. Los períodos de invierno más cortos, incrementan el potencial biótico de muchas poblaciones de parásitos. Un ejemplo de ello se observa en el ciclo biológico del crustáceo ectoparásito Argulus coregoni y el trematodo Diplostomum spathaceum, que son causa de serios problemas en la piscicultura de países del norte. (Rodríguez Diego, Olivares, Sánchez Castilleja, Alemán, \& Arece, 2013)

Las elevadas temperaturas podrían ampliar el rango de acción de los distintos reservorios y vectores de enfermedades transmisibles, entre los cuales se destacan roedores, mosquitos y garrapatas. Respecto a los mosquitos, algunos géneros se multiplican exponencialmente y aparecen en altitudes mayores, extendiéndose geográficamente con el riesgo consiguiente (Gorodner, 2016). Existe una fuerte sensación que el hallazgo de nuevos hábitats para especies de parásitos conocidos o relativamente desconocidos, hasta entonces naturales a otros situados posiblemente a grandes distancias, con una tendencia a ser aceptado como un fenómeno natural (Bulman \& Lamberti, 2011).

Pero no todo es lamento y desesperanza para la salud en América. Este continente a aportado también su fuerza de voluntad en crear vacunas y llevarlas a las poblaciones mas vulnerables produciendo que muchas enfermedades estén eliminadas.

Es importante recordar que las Américas tiene una historia de éxito en la eliminación de varias enfermedades prevenibles mediante la vacunación, entre ella a la viruela, la poliomielitis y el sarampión. Las Américas también lideran la eliminación de varias EID utilizando efectivamente la administración de medicamentos, el manejo intensivo de los casos, y mejorando las

\footnotetext{
479 Vol. 5, núm. 1, enero 2019, pp. 459-487
Nakin A. Véliz-Mero; Nidia E. Macías-Cedeño; José M. Piguave-Reyes; Yessenia L. MoreiraAndrade; Fabrizzio R. Araujo-Reyna; Mariela P. Sabando-Saltos
} 
condiciones sanitarias y ambientales. Como resultado de estos esfuerzos integrados, tres países de la región (Costa Rica, Surinam, Trinidad y Tobago) ya no se consideran más como países endémicos para la filariasis linfática; así como también, la eliminación de lepra como problema de salud pública a primer nivel administrativo nacional en 24 países endémicos en la región. (Ault, Catalá Pascual, Grados-Zavala, Gonzálvez García, \& Castellanos, 2014, pág. 324)

Como mitigar las enfermedades tropicales producto del cambio climático

Además de los factores que ahora favorecen las enfermedades transmitidas por vectores, como la variación estacional del tiempo, la situación socioeconómica, los programas de lucha anti vectorial, los cambios ambientales y la resistencia a los medicamentos, es muy probable que el cambio y la variabilidad climáticos influyan en la epidemiología de esas enfermedades. (Githeko, Lindsay, Confalonieri, \& Patz, 2009, pág. 32)

Estas enfermedades infecciosas y cuya epidemiología podrían afectarse en América por los cambios climáticos se pueden ver reflejada en la Tabla 2.

El poder contrarrestar esta epidemia productos del cambio climático requiere de un cambio en el paradigma de la sociedad donde esta sea la garante para que se construya una cultura en pro del desarrollo social de la mano con el fenómeno climatológico y sus causas.

Se debe entender el concepto de ecotono en epidemiología, como aquellas regiones de transición entre zonas endémicas y no endémicas donde la transmisión de una enfermedad puede ser inestable y quizá, por ende, puede existir incluso una mayor susceptibilidad a un impacto cuando se producen cambios climáticos considerables originándose entonces escenarios favorables para

\footnotetext{
480 Vol. 5, núm. 1, enero 2019, pp. 459-487 Nakin A. Véliz-Mero; Nidia E. Macías-Cedeño; José M. Piguave-Reyes; Yessenia L. MoreiraAndrade; Fabrizzio R. Araujo-Reyna; Mariela P. Sabando-Saltos
} 
la transmisión, con la ocurrencia de nuevas infecciones e incluso, en algunos casos de brotes epidémicos o epidemias. (Rodríguez-Morales, 2009, pág. 269)

Tabla 2. Enfermedades infecciosas y cuya epidemiología podría afectarse en América por los cambios climáticos

\begin{tabular}{lccc}
\hline \multicolumn{1}{c}{ ENFERMEDAD } & AGENTE & VECTOR & CLÍNICA \\
\hline Malaria & Plasmodium & Anopheles & Fiebres palúdicas \\
Leishmaniasis & Leishmania sp & Lutzomya & $\begin{array}{c}\text { Lesiones cutáneas, viscerales } \\
\text { y mucocutaneas }\end{array}$ \\
Dengue & virus & mosquitos & Fiebre hemorrágica \\
Hantavirosis & hantavirus & roedores & Fiebre hemorrágica \\
Fiebre amarilla & virus & mosquitos & Fiebre hemorrágica \\
Encefalitis de San Luis & Flavivirus & mosquitos & Encefalitis \\
Encefalitis equina venezolana & Arbovirus & mosquitos & Encefalitis \\
\hline
\end{tabular}

Fuente: (Sánchez, Mattar, \& González, 2009)

Este cambio cultural entre las sociedades, incluyendo dentro de los entes gubernamentales debe profundizarse con un cambio en las políticas educacionales desde varias aristas, pasando por la escuela hasta publicidad en los diferentes medios. Sin duda se debe profundizar este cambio cultural para poder así mitigar esta problemática que crece cada día más.

Control y vigilancia de enfermedades relacionadas con el clima a nivel estatal y regional; llevar a cabo evaluaciones de riesgo para entender las condiciones ambientales y climatológicas que favorecen la transmisión de enfermedades emergentes y reemergentes en diferentes zonas del país; desarrollar escenarios de cambio climático de alta resolución espacial y temporal; preparación para desastres; fortalecimiento de la lucha anti vectorial y de la higiene e inspección de los alimentos; provisión de suplementos nutricionales a poblaciones vulnerables; vacunación a 
todas las poblaciones vulnerables; atención a problemas de salud mental ante casos de desastre; y comunicación de riesgos ambientales. Aunado a lo anterior, es necesario tomar en consideración los factores influyentes adicionales, tales como los económicos, sociales, demográficos. (Moreno, 2010, pág. 168)

Existen diversas maneras de poder extrapolar esa cultura y es hacer hábitos ciudadanos en pro del bienestar social. La prevención del dengue clásico o hemorrágico, chikungunya, zica y otras enfermedades trasmitidas por mosquitos requiere la colaboración conjunta. Esta concientización de cómo controlar el mosquito que lo transmite además de básica y definitiva debe hacerse porque son los residentes, como posibles afectados, los únicos responsables de mantener sus patios y zonas aledañas inundadas, secas y libres de criaderos donde se puedan desarrollar los mosquitos (Rivera García, 2014).

Uno de los principales mosquitos, al cual se debe controlar, es el aedes aegypti el cual es el que proporciona el dengue, fiebre amarilla urbana, chikungunya y del zica. Tiene hábitos antropofílicos y domésticos. Los huevos son capaces de resistir desecación y temperaturas extremas con sobrevida de 7 meses a un año, las larvas se desarrollan entre $5^{\circ} \mathrm{C}$ a $29^{\circ} \mathrm{C}$, en 5 a 7 días (Maguiña Vargas, 2016).

Para la prevención del Dengue, fiebre Zika, fiebre Chikungunya y la fiebre amarilla, las personas pueden protegerse de las picaduras de mosquitos mediante el uso de repelentes, vistiendo camisas con mangas y pantalones largos. La eliminación de los criaderos donde se reproducen los mosquitos impide la propagación del virus. Ello conlleva a la eliminación de todo tipo de colector hídrico por pequeño que éste sea. El tratamiento inexcusable de lugares con acceso vedado es otro factor de suma importancia para el control vectorial. El Estado debiera disponer de medidas

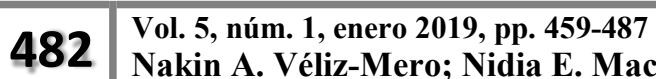

Nakin A. Véliz-Mero; Nidia E. Macías-Cedeño; José M. Piguave-Reyes; Yessenia L. Moreira-

Andrade; Fabrizzio R. Araujo-Reyna; Mariela P. Sabando-Saltos
} 
legales para superar dicha contingencia. Así también, debiera experimentarse la factibilidad de utilizar drones fumigadores. (Gorodner, 2016, pág. 32)

\section{Discusión y conclusiones.}

El paso evolutivo de la humanidad ha traído consigo el desarrollo de nuevas tecnologías que han permitido mejorar en la calidad de vida de las sociedades. Desde el mismo momento de la revolución industrial el crecimiento de la producción fue acelerado trayendo como consecuencia una ferviente precipitación de efectos colaterales que se están evidenciando en estas tres últimas décadas. La producción en serie ayuda a disminuir las necesidades, pero involucrando factores provenientes de la obsolescencia programada incrustada por las grandes empresas haciendo que las compras se aceleran y por ende la producción se incremente. Esta producción puede manifestarse en cualquier producto tanto material como alimentaria. La creación de alimentos transgénicos permite una cosecha más rápida, pero ocasiona también una emisión de gases que perjudican a la capa de ozono, ocurriendo el efecto de los gases invernadero. Estos GIV permiten un aceleramiento del cambio climático y por ende una variación de temperaturas las cuales traen consigo problemas que involucran todas las aristas de una sociedad cada vez creciente y demandante de estos productos.

Por supuesto estos GIV son catalizadores de muchas enfermedades por lo cual afectan la salud y la calidad de vida de la humanidad. Desde la movilización hacia otros sectores geográficos producto d ellos cambios climáticos que suceden a gran escala en muchas regiones, hasta la presencia en mayor cantidad de rayos UV provenientes del sol y debido al crecimiento aún mayor del orificio que se creó por esta contaminación. En definitiva, la salud ha venido en debacle y a aparecido nuevas y viejas enfermedades que se creían eliminadas hace 50 años o más. La movilización de la sociedad también trae consigo movilización de animales y entre ellos roedores y

\footnotetext{
483 Vol. 5, núm. 1, enero 2019, pp. 459-487
Nakin A. Véliz-Mero; Nidia E. Mac Nakin A. Véliz-Mero; Nidia E. Macías-Cedeño; José M. Piguave-Reyes; Yessenia L. MoreiraAndrade; Fabrizzio R. Araujo-Reyna; Mariela P. Sabando-Saltos
} 
mosquitos principales vectores que influyen directamente en la salud de la sociedad. Es por ello que se deben hacer planes para contrarrestar esta problemática.

Por lo tanto, estos planes tienen que ir de la mano entre las gobernantes y la sociedad. Primero para crear políticas y que estas sean cumplidas para que ayuden a mitigar el efecto de las enfermedades producto de este cambio climático y el otro factor es crear un cambio en la cultura produciendo u quiebre en viejos paradigmas. Muchos consideran que las soluciones a estos problemas son las viejas culturas indígenas, pero es necesario también involucrar esas nuevas tecnologías que ayuden a fomentar diferentes mecanismos que eviten el crecimiento de estas nuevas enfermedades tropicales. El dengue, chikungunya y el zica son enfermedades que nacen de una falta de educación de la sociedad pero que si se establece como cultura el prevenirlo entonces la mortalidad disminuye grandemente.

\section{Bibliografía.}

Ault, S. K., Catalá Pascual, L., Grados-Zavala, M. E., Gonzálvez García, G., \& Castellanos, L. (2014). El camino a la eliminación: un panorama de las enfermedades infecciosas desatendidas en América Latina y El Caribe. Revista Peruana de Medicina Experimental y Salud Pública, 31, 319-325.

Ballester, F. (2005). Contaminación atmosférica, cambio climático y salud. Revista Española de Salud Pública, 79, 159-175.

Barton, J. R. (2009). Adaptación al cambio climático en la planificación de ciudades-regiones. Revista de Geografia Norte Grande, (43), 5-30.

Berberian, G., \& Rosanova, M. T. (2012). Impacto del cambio climático en las enfermedades infecciosas. Archivos argentinos de pediatría, 110(1), 39-45.

Bianchi, E. \&. (2016). Empleo verde: el rol del estado y las empresas ante el cambio climático. Revista Argentina de Investigación en Negocios, 2(1-2), 7-20.

Blázquez, M. C. (2010). Cambio climático y salud. Informe SESPAS 2010. Gaceta Sanitaria, 24, 78-84. 
Bulman, G. M., \& Lamberti, J. C. (2011). Parásitos y enfermedades parasitarias emergentes y reemergentes: Calentamiento global, cambio climático, transmisión y migración de especies. evaluación de la participación del hombre. 1-15: Sitio Argentino de Producción Animal - Veterinaria Argentina, Bs. As., 28(282).

Butze Aguilar, W. (2004). El cambio climático: un problema de energía. El cotidiano, 19(123), 6679.

Cerda, J., Valdivia, G., Valenzuela, B., Teresa, M., \& Venegas, J. (2008). Cambio climático y enfermedades infecciosas: un nuevo escenario epidemiológico. Revista chilena de infectología, 25(6), 447-452.

Cueva, F. D. (2014). Efectos del cambio climático en la economía, el comercio internacional y la estrategia empresarial. Contabilidad y Negocios: Revista del Departamento Académico de Ciencias Administrativas, 9(18), 75-98.

Díaz Cordero, G. (2012). El cambio climático. Ciencia y sociedad. Volumen XXXVII, Número 2, 227-240.

Egea Jiménez, C. \&. (2011). Los desplazados ambientales, más allá del cambio climático. Un debate abierto. Cuadernos Geográficos, (49), 201-215.

Feo, O., Solano, E., Beingolea, L., Aparicio, M., Villagra, M., Prieto, M., . . . Silveti, R. (2009). Cambio climático y salud en la región andina. Revista Peruana de Medicina Experimental y Salud Pública, 26(1), 83-92.

Githeko, A. K., Lindsay, S. W., Confalonieri, U. E., \& Patz, J. A. (2009). El cambio climático y las enfermedades transmitidas por vectores: un análisis regional. Revista Virtual REDESMA, 3, 21., 21-38.

González Sánchez, Y. F., \& Gutiérrez Soto, T. (2013). El cambio climático y sus efectos en la salud. Revista Cubana de Higiene y Epidemiología, 51(3), 331-337.

Gorodner, J. O. (2016). Dengue, fiebre Zika y fiebre Chikungunya Patologías conminantes y cambio climático en América. Revista de la Asociación Médica Argentina, 129(1), 30-32.

HINNAWI, E. (1985). Environmental refugees. Nairobi: UNEP.

Iglesias, A. \&. (2009). Consecuencias del cambio climático para la agricultura:¿ un problema de hoy o del futuro? Revista española de estudios agrosociales y pesqueros, 221, 45-70.

Lampis, A. (2013). Vulnerabilidad y adaptación al cambio climático: debates acerca del concepto de vulnerabilidad y su medición. Cuadernos de Geografía, 22(2), 17-34.

López-Vélez, R., \& Molina Moreno, R. (2005). Cambio climático en España y riesgo de enfermedades infecciosas y parasitarias transmitidas por artrópodos y roedores. Revista Española de Salud Pública, 79, 177-190. 
Maguiña Vargas, C. (2016). Zika, la nueva enfermedad emergente en América: A new emerging disease in America. Revista Médica Herediana, 27(1), 3-6.

Martens, W. J. (1998). El cambio climático, la salud humana y el desarrollo sostenible. Revista Panamericana de Salud Pública, 4, 100-105.

Martín Vide, J. (2009). Conceptos previos y conceptos nuevos en el estudio del cambio climático reciente. Investigaciones Geográficas, $n^{\circ}$ 49, 51-63.

Más Bermejo, P. (2010). El cambio climático: un reto para los sistemas de salud. Revista Cubana de Higiene y Epidemiología, 48(3), 226-228.

Meira Cartea, P. Á. (2013). Problemas ambientales globales y educación ambiental: Una aproximación desde las representaciones sociales del cambio climático. Revista Integra Educativa, 6(3), 29-64.

Moreno, A. (2010). Efectos del cambio climático en la salud y los retos a enfrentar. En G. C. Delgado, C. Gay, M. Imaz, \& M. A. Martínez, México frente al cambio climático. (págs. 153-175). México, D.F.: Universidad Nacional Autónoma de México.

Pabón, J. D., \& Nicholls, R. S. (2005). El cambio climático y la salud humana. Biomédica, 25(1), 58.

Pecoul, B. \&. (2014). Enfermedades de la pobreza, enfermedades tropicales desatendidas. EutopíasVol. 7, 89-97.

Pinto Días, J. C. (1996). Las enfermedades tropicales y el enfoque de género. Bol Oficina Sanit Panam 121 (3), 260-281.

QUINTERO, M., CARVAJAL, Y., \& ALDUNCE, P. (2012). Adaptación a la variabilidad y el cambio climático: intersecciones con la gestión del riesgo. Revista Luna Azul, (34), 257-271.

Reyes-García, V. (2007). El conocimiento tradicional para la resolución de problemas ecológicos contemporáneos. Papeles de relaciones ecosociales y cambio global, 100, 109-116.

Rivera García, O. (2014). Aedes aegypti, virus dengue, chinkugunia, zika y el cambio climático. Máxima alerta médica y oficial. REDVET. Revista Electrónica de Veterinaria, 15(10), 1-10.

Rodríguez Diego, J. G., Olivares, J. L., Sánchez Castilleja, Y., Alemán, Y., \& Arece, J. (2013). Cambios climáticos y su efecto sobre algunos grupos de parásitos. Revista de Salud Animal, 35(3), 145-150.

Rodríguez-Morales, A. J. (2009). Cambio climático y salud humana: enfermedades transmisibles y América Latina. Revista Peruana de Medicina Experimental y Salud Pública, 26(2), 268269.

Sánchez, L. W. (2015). Análisis de las políticas públicas sobre el cambio climático en el Ecuador. Quito - Ecuador: Trabajo Final - Especialización en Derecho y Economía del Cambio Climático. 
Sánchez, L., Mattar, S., \& González, M. (2009). Cambios climáticos y enfermedades infecciosas: nuevos retos epidemiológicos. Revista MVZ Córdoba, 14(3), 1876-1885.

Sanz Donaire, J. J. (1999). Escepticismo al" cambio climático": el ejemplo de las temperaturas. NIMBUS $N^{\circ}$ 4, 173-198.

Sunyer, J. (2010). Promoción de la salud frente al cambio climático. Gac Sanit.2010;24(2):, 101102.

Valero-Bernal, M., \& Tanne, M. (2008). Globalización y salud: el caso de las enfermedades tropicales y olvidadas. Revista MVZ Córdoba, 13(1), 1252-1264.

Vargas, P. (2009). El cambio climático y sus efectos en el Perú. Lima: Banco Central de Reserva. Serie de Documentos de Trabajo, 1-59.

Vidal, V. (1999). Impactos de la aplicación de políticas sobre Cambio Climático en la forestación del páramo del Ecuador. Ecología política, (18), 49-53. 